\title{
Colaboração entre fornecedores de autopeças utilizando ferramentas da Indústria 4.0: uma revisão bibliométrica e sistemática da literatura
}

\section{Collaboration between Auto Parts Suppliers Using Resources of Industry 4.0: a bibliometric and systematic literature review}

Versão do autor aceita publicada online: 07 maio 2021

Publicado online: 30 jun. 2021

Como citar esse artigo - American Psychological Association (APA): Vasconcelos, I. C., \& Vanalle, R. M. (2021). Colaboração entre fornecedores de autopeças utilizando ferramentas da Indústria 4.0: uma revisão bibliométrica e sistemática da literatura. Exacta. DOI:

https://doi.org/10.5585/exactaep.2021.19489.

\section{Isabella Camargo Vasconcelos}

belinnh1@gmail.com

https://orcid.org/0000-0002-6155-298X

https://www.uninove.br/

Universidade Nove de Julho

Possui graduação em Administração de Empresas pela Universidade Paulista de Sorocaba (2018). Tem experiência profissional na área logistica e administrativo financeiro. Atualmente é Analista Administrativo Senior na Agência de Desenvolvimento e Inovação de Sorocaba (Parque Tecnológico de Sorocaba) e mestranda no Programa de Pós-Graduação em Engenharia de Produção da Universidade Nove de Julho - UNINOVE, Brasil.

Contato principal para correspondência.

\section{Rosangela Maria Vanalle}

rvanalle@uni9.pro.br

https://orcid.org/0000-0001-8265-0231

https://www.uninove.br/

Universidade Nove de Julho

Possui graduação em Engenharia de Produção Quimica pela Universidade Federal de São Carlos (1981), mestrado em Engenharia de Produção pela Universidade Federal do Rio de Janeiro (1987), doutorado em Engenharia Mecânica /Eesc pela Universidade de São Paulo (1995) e pós-doutorado pela Universidad Complutense de Madrid, 2006. Tem experiência na área de Engenharia de Produção, gerência da Produção, com ênfase em Sistemas de Produção, Estratégias e Organizações atuando principalmente nos seguintes temas: gestão da produção, gestão da cadeia de suprimentos e estratégia de produção.. Atualmente é Professora Pesquisadora do Programa de Pós-Graduação em Engenharia de Produção da Universidade Nove de Julho - UNINOVE, Brasil.

\section{Resumo}

Essa pesquisa busca a realização de uma revisão bibliométrica e sistemática da literatura para verificar o que se tem feito à respeito da Colaboração entre fornecedores de autopeças quanto aos aspectos da utilização das ferramentas disponíveis na Indústria 4.0, com o propósito de promover sugestões para pesquisas futuras e encontrar lacunas na literatura. 


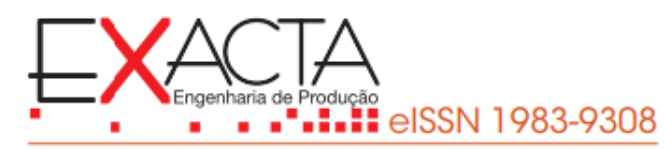

\section{Introdução}

A colaboração tem por definição o conjunto de atividades de diferentes empresas com a finalidade de atingir o objetivo individual de cada uma, caracterizado por um esforço entre duas ou mais organizações para alcançar resultados que não seriam possíveis isoladamente. É uma ação conjunta que envolve diversas etapas de produção de uma empresa (MORGAN; HUNT 1994).

Nas últimas décadas, houve a necessidade de empresas procurarem fora de suas organizações oportunidades de colaborar com parceiros para garantir que a Cadeia de Suprimentos seja eficiente e atenda às necessidades dinâmicas do mercado (FAWCETT; MAGNAN, 2004; LEJEUNE; YAKOVA, 2005).

A inclusão da $I o T$ na indústria faz parte da chamada Indústria 4.0, em que acontece a otimização de sistemas de manufatura e aplicações de novas tecnologias. Este conceito atualizado de Indústria faz com que a comunicação entre sistemas ocorra de formal efetiva, promovendo a gestão da produção (WOLLSCHLAEGER; SAUTER; JASPERNEITE, 2017).

O objetivo da IoT é formar uma infraestrutura digital para ajudar a interação das commodities, serviços e informações (LIU; SUN 2011).

A adoção de uma abordagem integrada em toda a cadeia de fornecimento requer uma troca entre autonomia e controle, para a qual o equilíbrio é único, entre cada relacionamento de parceiro de fornecimento. No ambiente industrial, onde novos modelos de negócios rapidamente suplantam os antigos, a inovação deve abranger mais do que as extensões das linhas de produção. Os atuais ambientes de negócios internacionais exigem inovação em todos os processos de negócios (GRAHAM; HARDAKER 2016).

Para diversas organizações, a colaboração é uma prática positiva quando comparado à realidade dos cooperados, contudo é destacado a heterogeneidade de concepções e práticas organizacionais (GUARDABASSIO; PEREIRA; DE AMORIM 2017).

Para garantir uma colaboração eficaz, os membros da cadeia são incentivados a definir claramente objetivos mútuos e medidas de desempenho associadas e vincular seus sistemas de desempenho à sincronização de decisões, compartilhamento de informações e alinhamento de incentivos de conhecimento. Uma ligação clara incentivará os membros da cadeia a melhorar os processos compartilhados da cadeia de suprimentos que beneficiam todos os membros (SIMATUPANG; SRIDHARAN, 2005).

Barratt (2004), identificou um significante número de elementos de colaboração, porém, segundo o autor, ainda não está claro como os elementos se inter-relacionam uns com os outros. Alguns destes elementos como a cultura da organização, confiança, troca de informação e as 
As redes de colaboração constituem arranjos colaborativos Inter organizacionais, que são de grande interesse nas organizações. Esses arranjos viabilizam e despertam o desenvolvimento de ações coletivas entre firmas, que são sustentados pela dinâmica colaborativa, tecnológica e social (CASTELLS 2010).

Para Penrose (1996) as diferenças sustentadas nos lucros das empresas podem ser atribuídas à diferença na aquisição de recursos. Os recursos e capacidades permitem que as empresas gerem rendas econômicas levando à sustentação de vantagem competitiva (AMIT; SCHOEMAKER 1993).

Para Oliveira Almeida (2017), a colaboração favorece a performance nacional ao alavancar a expansão e apoiar o crescimento e progresso das pequenas empresas.

Ide (2006) cita algumas tendências organizacionais proporcionadas pela colaboração, como estruturas organizacionais cada vez mais enxutas, ao eliminar atividades irrelevantes; redirecionamento do foco para o consumidor final; avanço nas evoluções de processos e globalização das ações das organizações a fim de encarar a concorrência.

Em um estudo realizado por Zylbersztajn (1994), houve uma avaliação das mudanças organizacionais ocasionadas por meio da utilização da Colaboração, e alguns resultados obtidos a partir da avaliação do estudo determinam um aumento da flexibilidade gerencial, maior capacidade de controle sobre os resultados, alocação eficiente dos recursos e aumento no número de consumidores.

\subsubsection{Colaboração na Cadeia de Suprimentos}

A Colaboração neste setor significa duas ou mais empresas autônomas trabalhando juntamente para organizar e realizar as operações na Cadeia de Suprimentos (SIMATUPANG; SRIDHARAN, 2002).

Diante de ambientes incertos, as empresas se esforçam para obter maior colaboração na cadeia de suprimentos para alavancar os recursos e o conhecimento de seus fornecedores e clientes (CAO;ZHANG, 2011).

A colaboração da cadeia de suprimentos (SCC) e a integração da cadeia de suprimentos (SCI) têm sido usadas de forma intercambiável, pois ambas se referem a um processo de acoplamento rígido entre colaboradores. No entanto, o termo integração significa o controle unificado (propriedade própria) de vários processos sucessivos ou similares para operações realizadas de maneira independente (WEBSTER, 1965; FLYNN et al., 2010).

Zhang et al. (2011), identifica, através da análise do impacto da cadeia de suprimentos colaborativa em vantagens e desempenho empresarial, um conjunto de sete dimensões interconectadas que compõem a colaboração eficaz da cadeia de suprimentos, sendo estes, a 
criação conjunta de conhecimentos, comunicação colaborativa, compartilhamento de recursos, alinhamento de incentivo, sincronização nas decisões, congruência nos objetivos e compartilhamento de informações.

Figura 2.1.1 - Impacto da Cadeia de Suprimentos Colaborativa em Vantagens e Desempenho

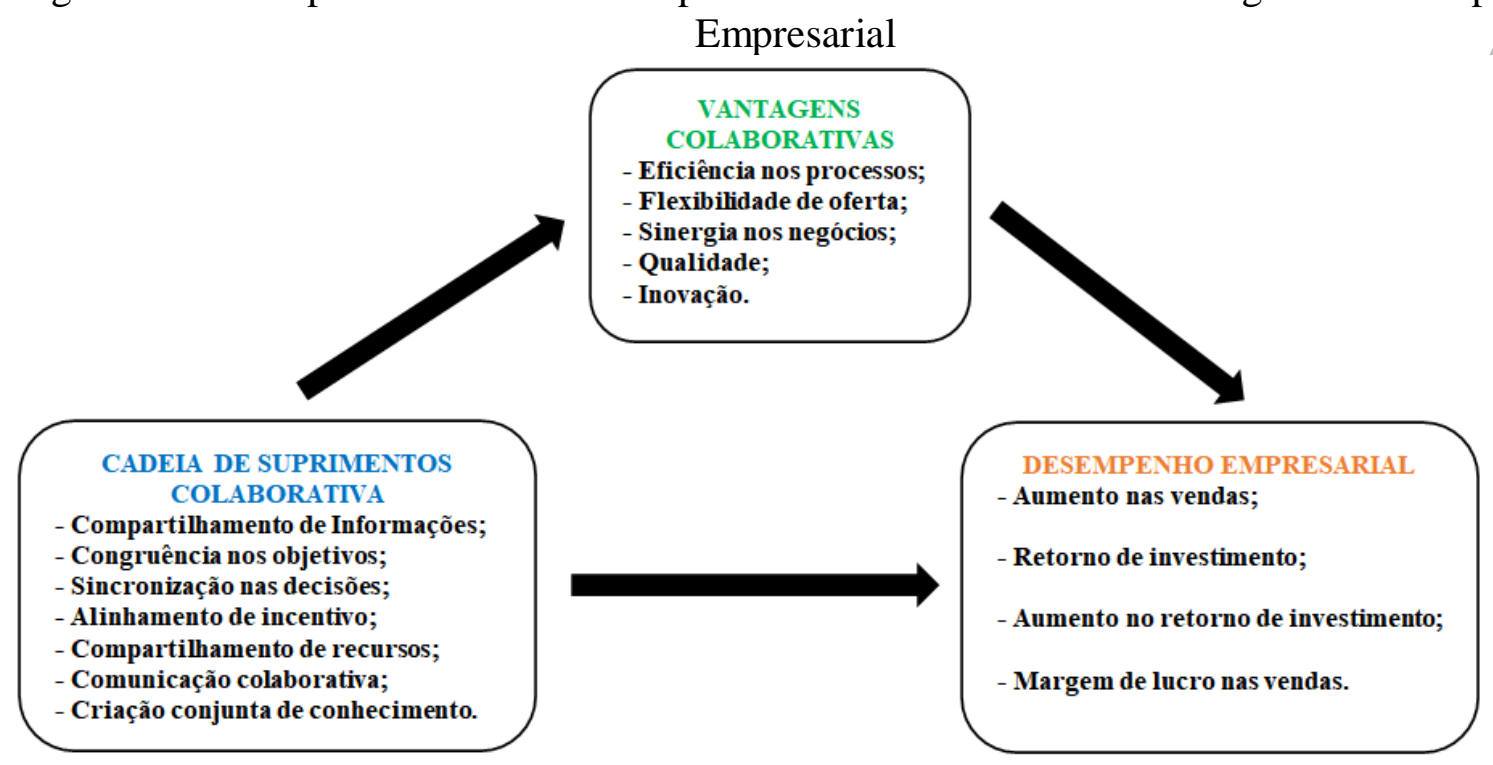

Fonte: adaptado de Zhang et al. (2011).

A colaboração na cadeia de suprimentos é definida como duas ou mais empresas trabalhando juntas para criar uma vantagem competitiva e lucros mais altos do que os que podem ser alcançados agindo sozinhos (Simatupang and Sridharan, 2002).

Este assunto foi conceituado de muitas maneiras diferentes por pesquisadores. No entanto, todas as pesquisas assumiram principalmente que a colaboração é um fenômeno unilateral que se concentra em um recurso específico da colaboração (Corbett et al., 2000; Lambert et al., 2003). A pesquisa baseada no processo da cadeia de suprimentos vê a colaboração como um esforço conjunto para redesenhar as operações da cadeia de suprimentos que resultam em melhor atendimento ao cliente e custos mais baixos (Hammer, 2001).

\subsection{Fornecedores de Autopeças}

Os Fornecedores de Autopeças e Montadoras concordam com as transformações mundiais que diariamente estão expostos, via abertura, aos novos padrões internacionais de concorrência. Os segmentos passam a ajustar-se aos novos padrões e os resultados são os processos de reestruturação no complexo automotivo do país (COSTA 1998). 


\subsection{Ferramentas da Indústria 4.0}

Assim como o conceito da Internet das Coisas, existem outras ferramentas e conceitos que compõem os aspectos essenciais da Indústria 4.0. Essas ferramentas são descritas nos pontos a seguir.

\subsubsection{Nuvem Compartilhada}

As nuvens são um grande aglomerado de recursos virtualizados, que são de fácil utilização e acesso. Os serviços da computação em nuvem permitem que indivíduos e companhias usufruam de softwares remotos de terceiros e componentes de hardware, permitindo que usem e mantenham recursos remotamente, com confiabilidade e baixo custo. Estes serviços fornecem uma interface abstrata que reúne recursos para obter uma utilização eficiente do sístema e concedem que os usuários expandam a escala para resolver problemas maiores, ao viabilizar que o software do sistema seja configurado conforme necessário para os requisitos individuais da aplicação (RAO, et al. 2012).

A computação em nuvem é um grande paradigma do futuro que disponibiliza muitas vantagens nos aspectos econômicos, como a economia de tempo, capacidades computacionais flexíveis, e poder de computação ilimitado. Os dados são transferidos, processados e armazenados por fornecedores de nuvem externos (KOK 2010).

\subsubsection{Análise Big Data}

A Big data é capaz de suportar coleta de dados em tempo real de fontes distintas, análise sistemática de dadôs e tomadas de decisões instantâneas, promovendo uma flexibilidade na manufatura, na qualidade da produção, eficiência energética e melhoria dos equipamentos através de uma manutenção antecipada (BAHRIN, et al. 2016).

A Análise Big Data baseia-se na agregação de conteúdo como informações comerciais, análise de mercado e ligações desconhecidas. Este conceito ajuda as empresas a obter tomada de decisão mais assertiva e na ocasião necessária (BORLIDO 2017).

\subsubsection{Impressão 3D e Soluções Robotizadas}

As Soluções Robotizadas são caracterizadas como sistemas flexíveis e autônomos que oferecem vantagem de custo, variedade de capacidade e realizam diversas tarefas nos processos da fábrica inteligente (PEI, et al. 2017).

Segundo Kamble et al. (2018), a impressão em 3D tem como base a manufatura aditiva, na qual forma os produtos finais através da construção de camadas sucessivas de materiais, evitando assim o processo de montagem. 


\subsubsection{Fabricação Digital}

A manufatura e conceito digital são atributos essenciais no desenvolvimento da Indústria 4.0, deste modo, a utilização de softwares de alta capacidade faz com que as instituições confiem nessa sólida transformação (BORLIDO 2017).

\subsubsection{Gerenciamento Remoto}

A IoT é capaz de permitir um gerenciamento remoto das operações da cadeira, melhor coordenação com parceiros e pode fornecer informações mais precisas para tomada de decisões mais eficazes (BEN DAYA; HASSINI; BAHROUN 2017).

\subsubsection{Monitoramento da Produção}

Através da correta e situada estrutura de serviços, o procedimento e comportamento da produção, pode ser carregado automaticamente e com eficiência, facilitando assim o Monitoramento da Produção (BÜYÜKÖZKAN; GÜLERYÜZ 2016).

A Indústria 4.0 permite a correta alocação, monitoramento e gerenciamento de recursos como: água, energia, matéria-prima e diversos outros consumíveis, através da interpretação e uso dos dados coletados em tempo real de produção, resultando em procedimentos sustentáveis (STRANGE; ZUCCHELLA 2016).

\subsubsection{Fábrica Inteligente}

A fábrica inteligente funciona com efetividade, já as máquinas inteligentes conectadas umas às outras, cooperam entre os funcionários, clientes e fornecedores, ou seja, de todos os stakeholders da indústria, promovendo assim, o dinamismo gerado para o autocontrole e cadeia analítica (BORLIDO 2017).

\subsubsection{RFID - Rastreabilidade Industrial}

A RFID é uma ferramenta tecnológica que ajuda a expandir a IoT. Esta utiliza ondas de rádio frequência para transmitir informações entre objetos selecionados, promovendo a automatização na identificação e comunicação entre as coisas (SHENG; LI; ZEADALLY 2008).

A RFID se comunica por tags (etiquetas). A antena do leitor cria um campo magnético juntamente com a antena da tag, e a partir disso, forma-se uma energia que é usada para mandar ondas ao leitor. Essas ondas são transformadas em informação eletrônica representando o código eletrônico do produto (ANGELES 2005). 
A RFID é conhecido como um dos principais sensores de conexão a objetos, capaz de transferir dados instantaneamente para processamento, monitoramento e controle em Cadeias de Suprimentos e têm sido amplamente estudado em gestão de estoques (ZHOU; Y.L.CHONG; W.T.NGAI 2015).

A IoT, com o auxílio das etiquetas de rádio frequência (RFID), é capaz de monitorar diversas ações, podendo assim ser muito utilizada no GCS (Gerenciamento da Cadeia de Suprimentos), controle de estoque, rastreamento de objetos na linha de produção e em processos. O uso de etiquetas é um dos fatores fundamentais visando benefícios e efetividade em vários processos. O número de empresas a procura da tecnologia por rádio frequência é crescente, pois elás esperam que tal tecnologia, possa impulsionar a forma de fechar negócios. A tecnologia vem sendo muito bem avaliada, pois consegue significantemente impulsionar o processo de negócio e destacar a performance organizacional (WU, et al. 2006).

A RFID monitora diversas funções, bem como a quantidade de produtos, prateleiras, data de vencimento, verificação para abastecimento das prateleiras, entre outras. No campo industrial, o RFID traz inúmeros benefícios para a organização, bem como a redução de refugo; maior visão da Cadeia de Suprimentos; colaboração entre parceiros comerciais e aumento das vendas (BARDAKI; KOUROUTHANASSIS; PRAMATARI 2012).

A RFID, vem sendo extensivamente utilizada para rastreamento de objetos em armazéns, no qual os produtos foram marcados com as etiquetas contendo as informações pertinentes e necessárias para controle e gestão do processo, sendo desta forma, de uso primordial na implantação e uso da IoT, gerando assim, um melhor controle de estoque e GCS (YAN, et al. 2017).

\subsubsection{Manutenção Inteligente}

Quanto aos níveis de manutenção, a configuração de sensores de alta qualidade, processadores entre outras tecnologias, trouxe a necessidade do desenvolvimento de sistemas inteligentes de suprimentos e análise de dados concernentes aos processos.

O departamento de manutenção é essencial para aproveitar todos os dados de qualidade, abastecida por sensores que possuem capacidade de gerenciar o planejamento do trabalho de maneira eficaz. Os recursos humanos devem estar dispostos para a revolução industrial pois o reconhecimento das informações precisas, irá cooperar com a empresa em vários sentidos (BORLIDO 2017). 


\subsubsection{Internet das Coisas}

A IoT pode ser descrita como a base para o desenvolvimento de serviços e aplicações que oferece o compartilhamento de informações entre os stakeholders da cadeia de suprimento provendo melhor visibilidade desta (BARDAKI; KOUROUTHANASSIS; PRAMATARI 2012)

A IoT caracteriza-se por ser um ótimo utensílio quanto a solução de questões concernentes à dados. Os autores Teimoury et.al (2013), verificaram questões que podem solucionar-se com a utilização de IoT e os categorizaram das seguintes formas: atraso para captar informações, lentidão ao acessar dados, espera no recebimento de uma resposta e atualização do sistema. Segundo Gershenfeld et.al (2004), a IoT tem feito e inspirado novas tendências e mudanças no gerenciamento das cadeias de suprimento, uma vez que utilizando tal conceito, os objetos e equipamentos terão seu próprio código de identificação, podendo compartilhar dados de forma autônoma.

Ku (2017), concluiu que a integração da IoT com as tecnologias da informação está habilitando a acelerada mudança tecnológica. $\mathrm{O}$ artigo explorou o que pode significar as tecnologias emergentes para armazenamento de dados e sua respectiva implementação em um alto volume usando o Zettabyte - ZB para Cadeias de Suprimento existentes em um mercado dinâmico para certos tipos de materiais críticos.

\section{Método de Pesquisa}

Essa pesquisa possui foco exploratório e natureza aplicada (Marconi \& Lakatos, 2010), com análise quantitativa e qualitativa (Kumar, 2011; Bryman, 1989).

O método aplicado no desenvolvimento da pesquisa foi a revisão bibliométrica (Cooper \& Lindsay, 1998), já que com os dados levantados é possível a avaliação de quantidades concernentes aos avanços científicos, além disso, com a revisão sistemática (Moher et al., 2009), verificamos o embasamento da investigação do tema sugeridos por Bardin (1986).

Neste trabalho, as revisões bibliométrica e sistemática da literatura foram empregadas para pesquisar o que se tem feito à respeito da Colaboração entre fornecedores de autopeças quanto aos aspectos da utilização das ferramentas disponíveis na Indústria 4.0, no intuito de identificar lacunas na literatura e promover opiniões para futuras pesquisas.

Com os dados da revisão bibliométrica, é possível encontrar aspectos de trabalhos utilizando dados quantitativos e gráficos (Pritchard, 1969), sendo que as revisões sistemáticas podem 
colaborar com o desenvolvimento do estudo de conteúdo para interpretar e fichar dados (Bardin 1986).

Como a pesquisa visa investigar o que se tem publicado sobre a Colaboração entre fornecedores de autopeças, quando se consideram as ferramentas disponíveis na Indústria 4.0, definiram-se as palavras-chave, bem como as bases de dados, delimitando-se assim os critérios para a busca dos artigos, conforme constam no Quadro 3.1.

Quadro 3.1 - Parâmetros de busca dos artigos

\begin{tabular}{|c|}
\hline CONJUNTO/ PALAVRAS \\
\hline $\begin{array}{l}\text { Collaboration "OR" Collaborator OR" Cooperator "OR" Partners "OR" Collaborative } \\
\text { "AND" } \\
\text { Suppliers "OR" Supply "OR" Distributor } \\
\text { "AND" } \\
\text { Auto parts }\end{array}$ \\
\hline $\begin{array}{l}\text { Collaboration "OR" Collaborator OR" Cooperator "OR" Partners "OR" Collaborative } \\
\text { "AND" } \\
\text { Suppliers "OR" Supply "OR" Distributor } \\
\text { "AND" } \\
\text { Auto parts } \\
\text { "AND" } \\
\text { Industrie } 4.0 \text { "OR" Industry } 4.0\end{array}$ \\
\hline $\begin{array}{l}\text { Suppliers "OR" Supply "OR" distributor } \\
\text { "AND" } \\
\text { Auto parts } \\
\text { "AND" } \\
\text { Industrie } 4.0 \text { "OR" Industry } 4.0\end{array}$ \\
\hline $\begin{array}{l}\text { Collaboration "OR" Collaborator OR" Cooperator "OR" Partners "OR" Collaborative } \\
\text { "AND" } \\
\text { Industrie } 4.0 \text { "OR" Industry } 4.0\end{array}$ \\
\hline
\end{tabular}
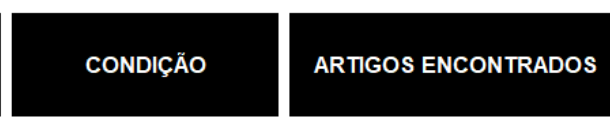

\begin{tabular}{|c|}
\hline TITULO/ TITULO \\
\hline ASSUNTOIASSUNTO \\
\hline TITULOI ASSUNTO \\
\hline ASSUNTO / TITULO \\
\hline
\end{tabular}

\begin{tabular}{|c|}
\hline 1 \\
\hline 42 \\
\hline 10 \\
\hline 4 \\
\hline
\end{tabular}

\begin{tabular}{|c|}
\hline TITULO/ TITULO \\
\hline ASSUNTOIASSUNTO \\
\hline TITULOI ASSUNTO \\
\hline ASSUNTO / TITULO \\
\hline
\end{tabular}

\begin{tabular}{|c|}
\hline 0 \\
\hline 0 \\
\hline 0 \\
\hline 0 \\
\hline
\end{tabular}

\begin{tabular}{|c|}
\hline TITULO/ TITULO \\
\hline ASSUNTOIASSUNTO \\
\hline TITULOI ASSUNTO \\
\hline ASSUNTO / TITULO \\
\hline
\end{tabular}

\begin{tabular}{|c|}
\hline 0 \\
\hline 1 \\
\hline 0 \\
\hline 0 \\
\hline
\end{tabular}

\begin{tabular}{|c|}
\hline TITULO/ TITULO \\
\hline ASSUNTO/ASSUNTO \\
\hline TITULOI ASSUNTO \\
\hline ASSUNTO / TITULO \\
\hline
\end{tabular}

\begin{tabular}{|c|}
\hline 31 \\
\hline 565 \\
\hline 107 \\
\hline 209 \\
\hline
\end{tabular}

Fonte: elaborado pelos autores.

Segundo Bardin (1986), através da definição do problema de pesquisa, é possível realizar a seleção de trabalhos que possam colaborar com a pesquisa, assim, as seguintes questões foram levantadas para guiar a pesquisa:

Q1: Existe colaboração entre fornecedores de autopeças?

Q2: Caso haja a colaboração entre fornecedores de autopeças, quais são as ferramentas utilizadas da Indústria 4.0?

Q3: Quais são os principais benefícios e desafios dessa colaboração pelo uso das ferramentas da Indústria 4.0. 
A pesquisa abordou um composto de critérios de exclusão e inclusão de trabalhos que se baseassem no assunto do documento em que aponta meta-analises (PRISMA) e revisões sistemáticas, constituído por Moher et al. (2009).

Figura 3.1 - Diagrama de seleção e avaliação dos artigos

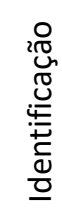

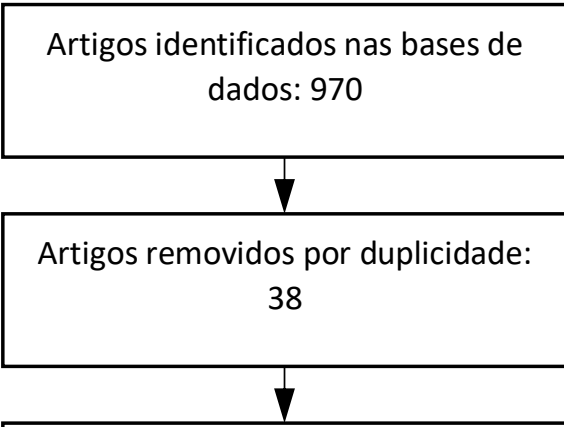

Artigos restantes: 932

\begin{tabular}{l}
$\frac{0}{0}$ \\
$\frac{\pi}{0}$ \\
$\frac{0}{\overline{0}}$ \\
\hline$\frac{0}{00}$ \\
$\frac{0}{4}$
\end{tabular}

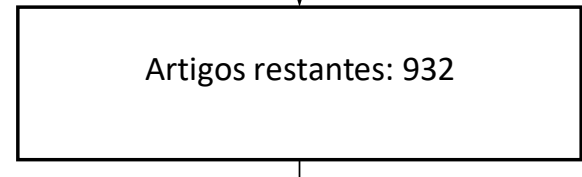

Artigos completos avaliados para elegibilidade: 932

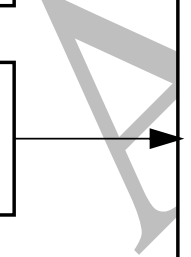

Foram excluídos artigos que não traziam informações pertinentes ao assunto que se deseja estudar, ou seja, A Colaboração entre Fornecedores de Autopeças Utilizando Ferramentas da Industria 4.0 (606) ou que não mencionavam $A$ Colaboração entre Fornecedores de Autopeças (303).

Fonte: adaptado de Moher et al. (2009).

A Figura 3.1, demonstra o PRISMA (Moher et al., 2009) que relata sobre as pesquisas em bases de dados resultaram em 970 artigos encontrados, sendo que, após a exclusão de artigos repedidos, sobraram 932 para a análise. Com a leitura completa dos artigos, considerando-se os parâmetros para exclusão exibidos na Figura 3.1, foram considerados apropriados para o desenvolvimento da pesquisa, 23 artigos conforme evidenciado na Figura 3.2. 
Figura 3.2 - Captura da Pasta com os Artigos Relevantes

\begin{tabular}{|c|c|c|}
\hline 01- A causal model for supply chain partner's ... & Adobe Acrobat D... & $356 \mathrm{~KB}$ \\
\hline 02 - An integrative framework for supply chai... & Adobe Acrobat D... & $281 \mathrm{~KB}$ \\
\hline 03 - Automobile industry, guanxi, and social n... & Adobe Acrobat D... & $181 \mathrm{~KB}$ \\
\hline 04 - Building co-operative supply chain in aut... & Adobe Acrobat D... & $152 \mathrm{~KB}$ \\
\hline 05 - Collaboration between competitors - Ca... & Adobe Acrobat D... & $312 \mathrm{~KB}$ \\
\hline 06 - Collaboration mechanisms to increase pr... & Adobe Acrobat D... & $615 \mathrm{~KB}$ \\
\hline 07 - Collaborative design procedure for suppl... & Adobe Acrobat D... & $49.185 \mathrm{~KB}$ \\
\hline 08 - Collaborative new product development ... & Adobe Acrobat D... & $50 \mathrm{~KB}$ \\
\hline 09 - Collaborative supply chain management ... & Adobe Acrobat D... & $257 \mathrm{~KB}$ \\
\hline 10 - Comprehensive evaluation of the auto $\mathrm{p} . .$. & Adobe Acrobat D... & $476 \mathrm{~KB}$ \\
\hline 11 - Effect of supply chain strategic collabora... & Adobe Acrobat D... & $215 \mathrm{~KB}$ \\
\hline 12 - Evaluation of enterprise cooperation thr... & Adobe Acrobat D... & $147 \mathrm{~KB}$ \\
\hline 13 - Identifying and modeling barriers to coll... & Adobe Acrobat D... & $191 \mathrm{~KB}$ \\
\hline 14 - Information sharing in supply chain colla... & Adobe Acrobat D... & $726 \mathrm{~KB}$ \\
\hline 15 - Networking with Partners Is Essential for I... & Adobe Acrobat D... & $234 \mathrm{~KB}$ \\
\hline 16 - Performance Evaluation of Auto Parts Su... & Adobe Acrobat D... & $59.480 \mathrm{~KB}$ \\
\hline 17 - Sales-forecast-based auto parts multiple... & Adobe Acrobat D... & $53.715 \mathrm{~KB}$ \\
\hline 18 - Supply chain collaboration and logistical... & Adobe Acrobat D... & $117 \mathrm{~KB}$ \\
\hline 19 - Supply chain collaboration in the presen... & Adobe Acrobat D... & $1.356 \mathrm{~KB}$ \\
\hline 20 - Supply chain collaboration Impact on col... & Adobe Acrobat D... & $602 \mathrm{~KB}$ \\
\hline 21 - Supply chain collaboration whats happe... & Adobe Acrobat D... & $148 \mathrm{~KB}$ \\
\hline 22 - The effects of supply chain collaboration... & Adobe Acrobat D... & $1.061 \mathrm{~KB}$ \\
\hline 23 - Understanding the meaning of collabora... & Adobe Acrobat D... & $400 \mathrm{~KB}$ \\
\hline
\end{tabular}

Fonte: elaborado pelos autores.

Não houve limitação em relação à data de início de publicações, porém, a data final foi setembro de 2020. Thomé et. al (2016), explica que é imprescindível a interrupção da data para toda análise bibliográfica, isso ocorre pois o ano de 2020 ainda não havia se encerrado durante a pesquisa, portanto, 2020 foi considerado um ano inteiro, devido à necessidade da conclusão do artigo.

Com a seleção dos artigos, a avaliação iniciou-se com procedimentos bibliométricos que faziam parte da cienciotometria, considerada para encontrar a performance científica baseada no tema das publicações (Godin 2006; Verbeek et al. 2002), além disto, outro aspecto analisado foi a evolução quantificável do campo conhecimento atual (RAMOS-RODRIGUEZ;RUIZNAVARRO 2004).

Foram atribuídas na mesma lógica o agrupamento de artigos por ano de publicação, para evidenciar a importância dessa pesquisa (Andres, 2009); o conjunto de artigos por periódico, para avaliar os que mais divulgam sobre a integração das ferramentas da indústria 4.0 à colaboração entre fornecedores de autopeças, pois, de acordo com Akmal et al. (2018), a 
investigação da quantidade de artigos por periódicos é excelente para os trabalhos de pesquisadores sejam direcionados a apropriados periódicos; o número de publicações por países identifica em quais países existem mais publicações sobre o assunto pesquisado; e, por fim, as metodologias usadas nos artigos pesquisados, para saber a maneira como este assunto está sendo investigado e poder indicar como pesquisas futuras as metodologias menos trabalhadas (OLIVEIRA NETO et al., 2018).

A revisão sistemática foi conduzida em seguida com base na principal questão que se refere à Colaboração entre fornecedores de autopeças utilizando as ferramentas da indústria 4.0, foi elaborado então, um relatório com trabalhos que consideram um ou mais aspectos da colaboração entre fornecedores de autopeças. Também foi considerada nesse levantamento a maneira como esses fornecedores se colaboram, ou seja, se utilizam alguma ferramenta disponível na indústria 4.0.

Em seguida, como não foi identificado a colaboração entre fornecedores utilizando ferramentas da indústria 4.0, também foi feita uma análise no sentido de identificar como os fornecedores de autopeças colaboram embora sem, ainda, utilizar as ferramentas da Industria 4.0 para determinada colaboração.

Por fim, foi analisada a concentração dos aspectos da Indústria 4.0 por tecnologias. Tudo isso foi analisado para tentar identificar se os fornecedores de autopeças utilizavam alguma ferramenta disponível na Industria 4.0 para colaborarem entre si. De tal modo, foi possível ressaltar direções aos pesquisadores para a evolução de pesquisas futuras, como indicado por Rocha et al. (2012) e Oliveira Neto et al. (2018).

\section{Resultados e Discussões}

Os resultados das análises a seguir abordam o aspecto bibliométrico do presente estudo. $\mathrm{O}$ Gráfico 4.1 apresenta a relação dos países dos 23 artigos selecionados na presente pesquisa. A região com predominância dos artigos/ estudos encontrados foi os Estados Unidos da América (7 artigos), seguido pela China (6 artigos). 
Gráfico 4.1 - Artigos por Países

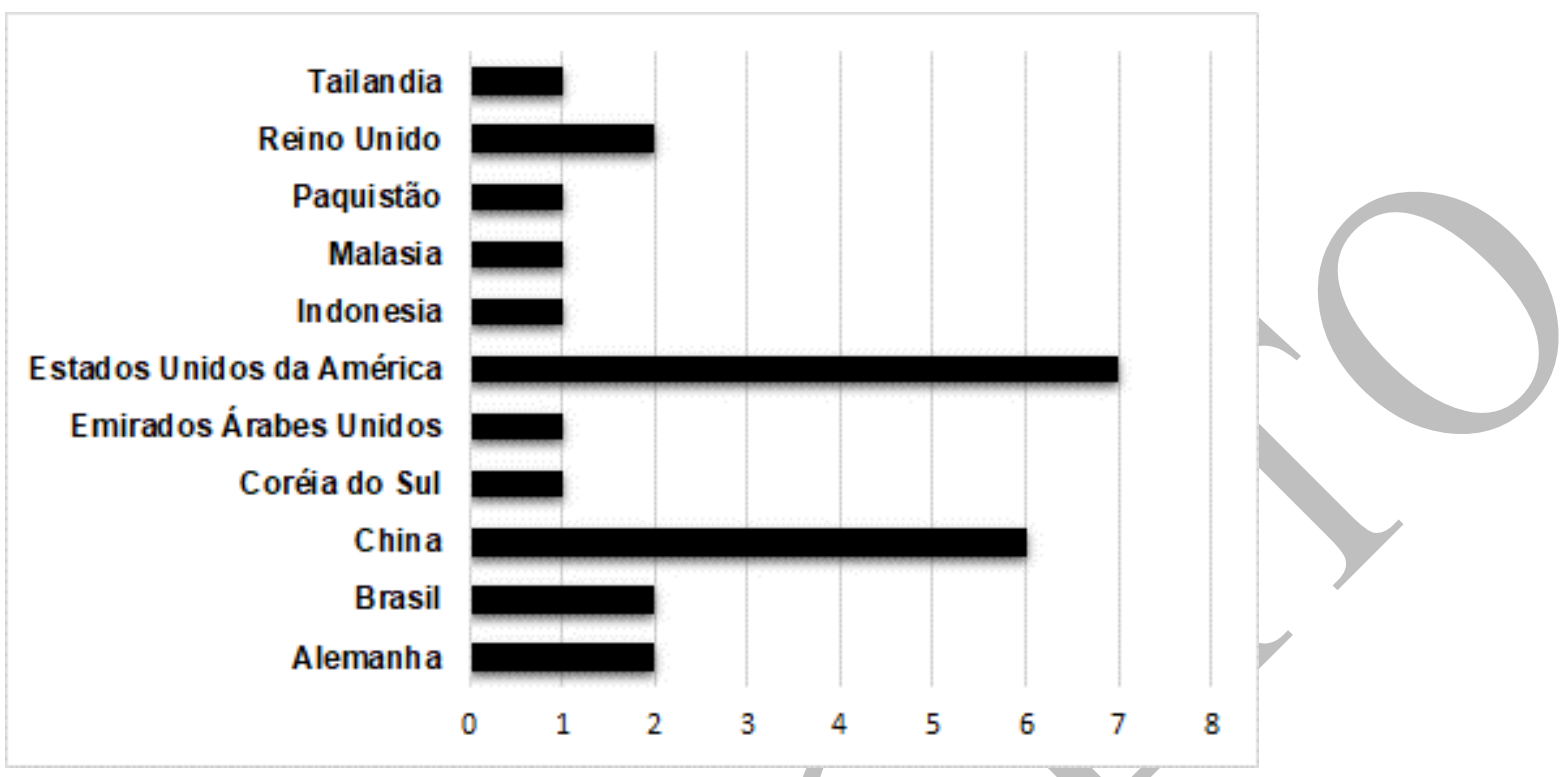

Fonte: elaborado pelos autores.

O gráfico da evolução das publicações dos artigos que abordam o assunto pesquisado mostra algumas oscilações, o ano em que mais houve publicações relevantes ao tema (3 publicações em cada ano) foi 2001, 2005, 2013 e 2020.

Gráfico 4.2 - Artigos por ano de publicação

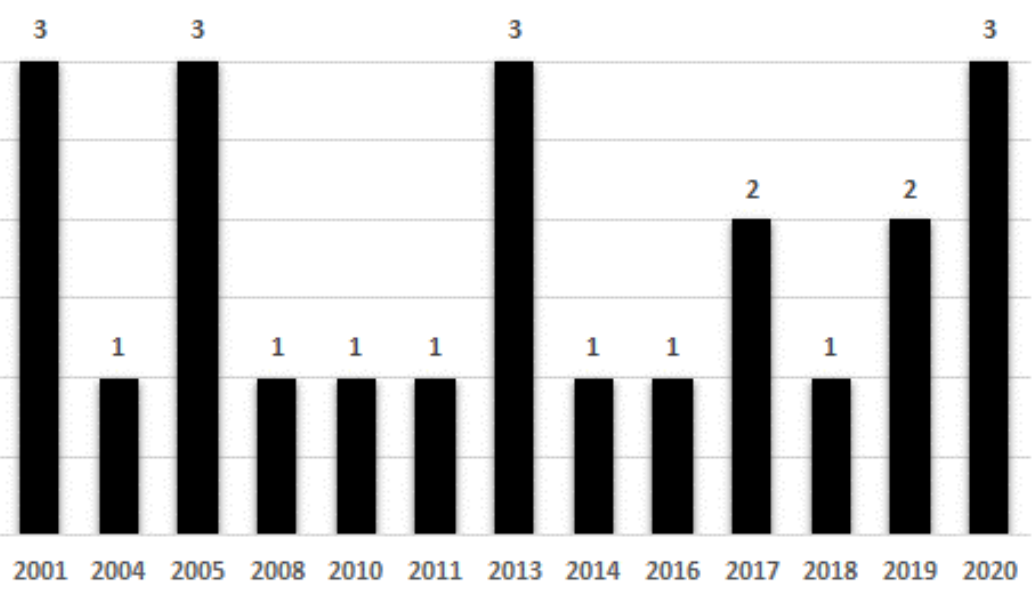

Fonte: elaborado pelos autores.

Alguns artigos pesquisados propuseram um modelo de pesquisa e outros buscavam evidenciar determinados comportamentos ou indicadores. De certa forma, observa-se certa vantagem entre os artigos pesquisados quanto à natureza da pesquisa, sendo quantitativo a porcentagem de $39 \%$ e as pesquisas qualitativas $31 \%$. 
Gráfico 4.3 - Natureza da Pesquisa

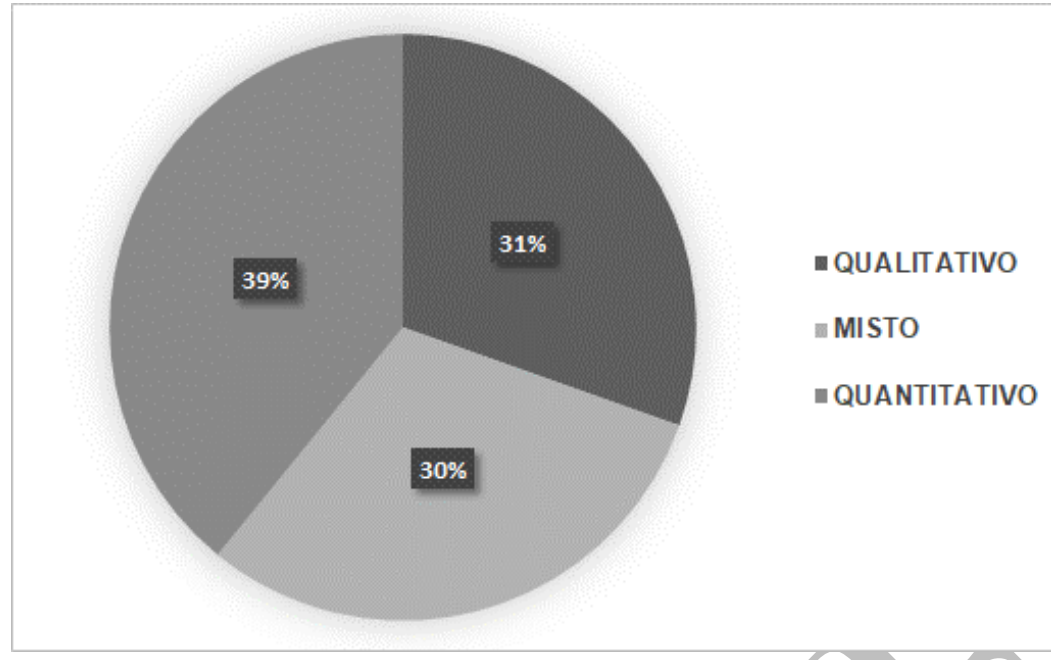

Fonte: elaborado pelos autores.

Os tipos de artigos dos quais foram obtidos e usados para execução dos trabalhos, seguem a disposição conforme o gráfico e tabela abaixo:

\section{Gráfico 4.5 - Tipos de Artigos}

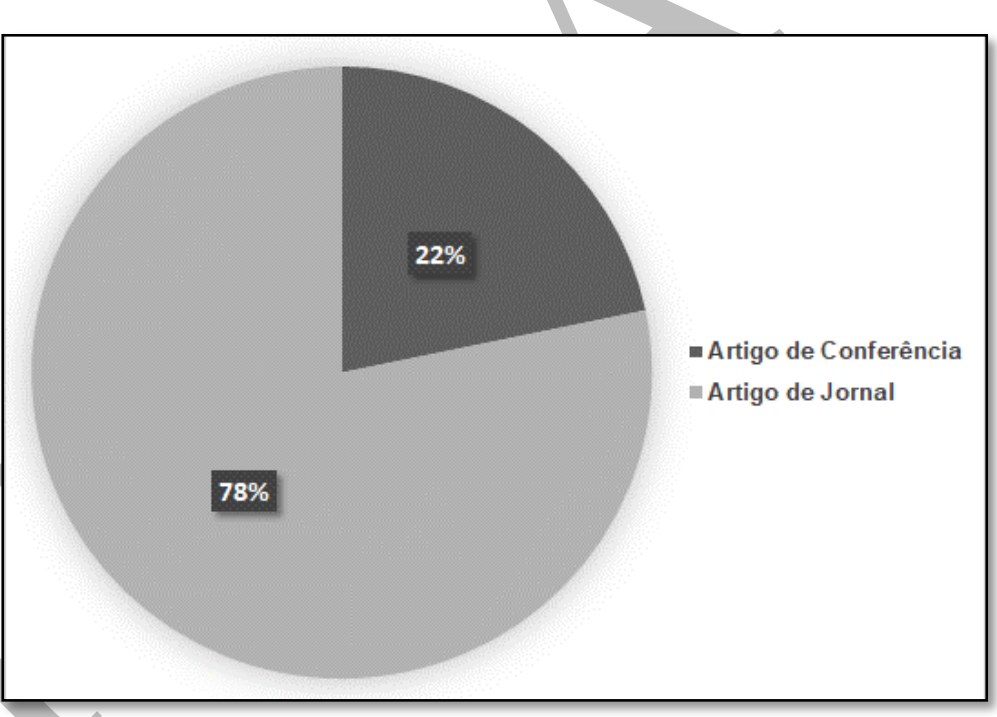

Fonte: elaborado pelos autores. 
Tabela 4.1: Artigos encontrados por periódicos

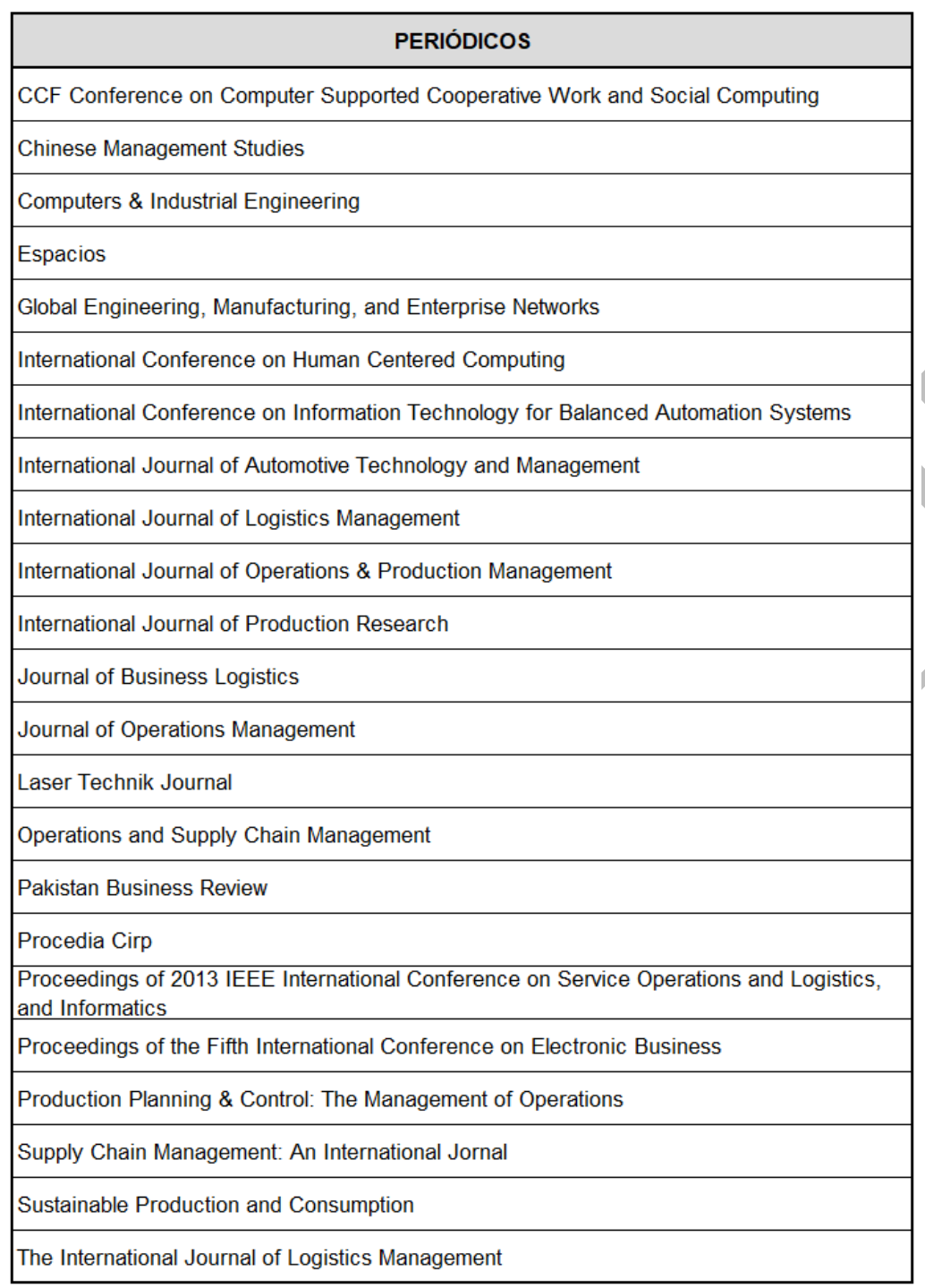

Fonte: elaborado pelos autores.

Dentre os artigos pesquisados, merece destaque o artigo de Cao e Zhang (2011), que identificaram um conjunto de sete dimensões interconectadas que compõem a colaboração eficaz da cadeia de suprimentos: Compartilhamento de informações, Congruência de objetivos, Sincronização de decisões, Alinhamento de incentivos, Compartilhamento de recursos, Comunicação colaborativa e a Criação conjunta de conhecimento. Segundo estes autores, os benefícios da colaboração serão alcançados quando todas as partes da cadeia de suprimentos, dos fornecedores aos clientes, cooperarem, pois a criação conjunta de um ritmo comum de compartilhamento de informações, reabastecimento e sincronização de suprimentos em uma cadeia de suprimentos pode reduzir o excesso de estoques, evitar o caro efeito de chicote, melhorar a sinergia nos negócios, melhorar a qualidade nos negócios, oferecer flexibilidade e inovação conjunta. 
Outro artigo que merece destaque, discute sobre uma estrutura com cinco recursos pelos quais os membros da cadeia se envolvem na definição de ações específicas a serem executadas em colaboração. Para uma colaboração ativa, os membros da cadeia são estimulados a determinar objetivos em comum, bem como medidas de atuação adjuntas e acoplar seus sistemas de atuação à sincronização de disposições, compartilhamento de informações e alinhamento de estímulos de conhecimento. Uma conexão evidente estimulará os membros da cadeia a aperfeiçoar os processos compartilhados da cadeia de suprimentos que trarão benefícios aos envolvidos (SIMATUPANG; SRIDHARAN, 2005).

O artigo de Barratt (2004), mostra uma expressiva quantidade de elementos de colaboração, porém, segundo o autor, ainda não está claro a maneira como os elementos se integram. Determinados elementos como a cultura da organização, confiança, troca de informação e as medidas de desempenho da cadeia de suprimentos constituíram em boas partes desconhecidas por sua complexidade e precisam de precaução singular em termos de ānálises futuras.

\section{Conclusões}

De acordo com os resultados da pesquisa, fica evidente que o tema Colaboração entre Fornecedores já vem sendo tratado. Os autores mostraram varias formas de utilizar a colaboração dentro das empresas, mais especificamente dentro da Cadeia de Suprimentos e entre Fornecedores de Autopeças. A principal lacuna percebida foi a falta de trabalhos que remetem a Colaboração entre os Fornecedores de Autopeças Utilizando Ferramentas da Indústria 4.0. Alguns artigos apresentaram benefícios da Colaboração entre stakeholders da Cadeia de Suprimentos, porém, sem relatar nenhuma informação do uso das ferramentas da Indústria 4.0. Sugere-se como pesquisa futura, trabalhos que investiguem a utilização das ferramentas da Indústria 4.0 por fornecedores de autopeças para promoção da Colaboração que demonstra ser benéfica às empresas.

\section{REFERÊNCIAS}

Akmal, A., Podgorodnichenko, N., Greatbanks, R., \& Everett, A. M. (2018). Bibliometric analysis of production planning and control (1990-2016). Production Planning \& Control, 29(4), 333-351.

Andrés, A. (2009). Measuring academic research: How to undertake a bibliometric study. Elsevier. 
Angeles, R. (2005). RFID technologies: supply-chain applications and implementation issues. Information systems management, 22(1), 51-65.

Amit, R., \& Schoemaker, P. J. (1993). Strategic assets and organizational rent. Strategic management journal, 14(1), 33-46.

Bardin, L. (1986). El anàlisis de contenido. [s.l.] Madrid. Ediciones Akal, Spain.

Bardaki, C., Kourouthanassis, P., \& Pramatari, K. (2012). Deploying RFID-enabled services in the retail supply chain: Lessons learned toward the internet of things. Information Systems Management, 29(3), 233-245.

Barney, J. (1991). Firm resources and sustained competitive advantage. Journal of management, 17(1), 99-120.

Bahrin, M., Othman, N. A. M., Talib, M. Industry 4.0: A review on industrial automation and robotic. 2016.

Barratt, M. (2004). Understanding the meaning of collaboration in the supply chain. Supply Chain Management: an international journal.

Ben-Daya, M., Hassini, E., \& Bahroun, Z. (2019). Internet of things and supply chain management: a literature review. International Journal of Production Research, 57(15-16), 4719-4742.

Borlido, D. J. A. (2017). Indústria 4.0: Aplicação a Sistemas de Manutenção. U. Porto FEUP Faculdade de Engenharia Universidade do Porto.

Bryman, A. (1989). Research methods and organization studies. London: Unwin Hyman.

Büyüközkan, G., \& Güleryüz, S. (2016). Multi criteria group decision making approach for smart phone selection using intuitionistic fuzzy TOPSIS. International Journal of Computational Intelligence Systems, 9(4), 709-725. 
Da Rocha, A. C., Camargo, C. R., Kneipp, J. M., Ávila, L. V., Gomes, C. M., \& Madruga, L. R. D. R. G. (2012). Gestão para a sustentabilidade na indústria mineral: Um levantamento da produção científica e dos hot topics publicados na última década. Estudos do CEPE, 228-251.

Rao, B. P., Saluia, P., Sharma, N., Mittal, A., \& Sharma, S. V. (2012, December). Cloud computing for Internet of Things \& sensing based applications. In 2012 Sixth International Conference on Sensing Technology (ICST) (pp. 374-380). IEEE.

Sheng, Q. Z., Li, X., \& Zeadally, S. (2008). Enabling next-generation RFID applications: Solutions and challenges. Computer, 41(9), 21-28.

Siemens. Profinet With Step7 . Vol. 13. 2014.

Simatupang, T. M., \& Sridharan, R. (2005). An integrative framework for supply chain collaboration. The international journal of logistics management.

Simatupang, T. M., \& Sridharan, R. (2002). The collaborative supply chain. The international journal of logistics management, 13(1), 15-30.

Strange, R., \& Zucchella, A. (2017). Industry 4.0, global value chains and international business. Multinational Business Review.

Teimoury, E., Fathian, M., \& Chambar, I. (2013). Automation of the supply chain performance measurement based on multi-agent system. International Journal of Agile Systems and Management, 6(1), 25-42.

Thomé, A. M. T., Scavarda, L. F., \& Scavarda, A. J. (2016). Conducting systematic literature review in operations management. Production Planning \& Control, 27(5), 408-420.

Verbeek, A., Debackere, K., Luwel, M., \& Zimmermann, E. (2002). Measuring progress and evolution in science and technology-I: The multiple uses of bibliometric indicators. international Journal of management reviews, 4(2), 179-211. 
Webster Jr, F. E. (1965). The deal-prone consumer. Journal of Marketing Research, 2(2), 186189.

Wollschlaeger, M., Sauter, T., \& Jasperneite, J. (2017). The future of industrial communication: Automation networks in the era of the internet of things and industry 4.0. IEEE industrial electronics magazine, 11(1), 17-27.

Wu, N. C., Nystrom, M. A., Lin, T. R., \& Yu, H. C. (2006). Challenges to global RFID adoption. Technovation, 26(12), 1317-1323.

Yan, B., Jin, Z., Liu, L., \& Liu, S. (2018). Factors influencing the adoption of the internet of things in supply chains. Journal of Evolutionary Economics, 28(3), 523-545.

Zhou, L., Chong, A. Y., \& Ngai, E. W. (2015). Supply chain management in the era of the internet of things. International Journal of Production Economics, 159, 1-3.

Zylbersztajn, D. (1994). Organização de cooperativas: desafios e tendências. Revista de Administração, 29(3), 23-32. 